\title{
Radical Classes Closed Under Products
}

\author{
Barry J. Gardner
}

\begin{abstract}
This is a survey of what is known about Kurosh-Amitsur radical classes which are closed under direct products. Associative rings, groups, abelian groups, abelian $\ell$-groups and modules are treated. We have attempted to account for all published results relevant to this topic. Many or most of these were not, as published, expressed in radical theoretic terms, but have consequences for radical theory which we point out. A fruitful source of results and examples is the notion of slenderness for abelian groups together with its several variants for other structures. We also present a few new results, including examples and a demonstration that $e$-varieties of regular rings are product closed radical classes of associative rings.
\end{abstract}

\section{Introduction}

Which radical classes are closed under (direct) products? This is a very natural question, but one for which we have at present nothing remotely like an answer. In particular we know nothing about the sorts of classes that define product-closed lower radical classes and nothing about the semi-simple classes corresponding to product-closed radical classes.

Although product-closure has not been studied systematically in full generality by radical theorists, there is a substantial collection of results in the literature concerned with aspects of it, not all of which come from studies with an overtly radical-theoretic motivation.

Key Words: Radical class, product, slender, ring, group, module

2010 Mathematics Subject Classification: Primary 16N80, 16S90, 18E40, 20M11; Secondary $08 \mathrm{~A} 15$.

Received: April, 2012.

Revised: May, 2012.

Accepted: February, 2013. 
Semi-simple radical classes and more generally (in some cases) radical classes which are varieties are product-closed. These were dealt with quite thoroughly in the 70s and 80s of last century and will be barely touched on here. They are covered quite thoroughly in the survey [32] and its references.

For a class $X$ of groups a group is $X$-perfect if it has no non-trivial homomorphic images in $X$. (For $X$ the class of abelian groups we get perfect groups in the more familiar sense.) Quite a lot is known about classes $X$ for which the class of $X$-perfect groups is closed under products. Sometimes the latter are radical classes.

Hereditary product-closed radical classes of modules ( $T T F$-classes) have been extensively studied. (For modules they are the semi-simple radical classes adverted to above.)

In abelian groups there are no non-trivial product-closed hereditary radical classes, but here product-closure has been studied in a context more general than (Kurosh-Amitsur) radical theory: the commutation of subfunctors of the identity with products. Here we find, among other things, examples of radical classes closed under products of some sizes (e.g. products of countably many abelian groups) but not arbitrary ones. Many results in this area depend on "extra" set-theoretic axioms, but this has minimal impact on what we deal with.

The classes of $(\rho ; \sigma)$-regular rings introduced by McKnight and Musser [58] are product closed radical classes.

We shall deal with all the foregoing in some detail. A few new related results and examples will also be presented, including a (negative) result from abelian $\ell$-groups which provides some contrast with abelian groups.

The notion of an $e$-variety originated in semigroup theory [44], [54], and is adapted easily to regular rings [46]. An e-variety of regular rings is a nonempty class closed under homomorphic images, products and regular subrings (so varieties of strongly regular rings are examples). We'll show that all these classes are radical classes.

The known results involving product-closed radical classes are a disparate lot; a slender thread which connects some of them is the notion of a slender (abelian) group due to Łoś. This notion was extended to groups by Göbel [41]. Various radical classes of groups and of abelian groups which are closed under products where the number of factors is non-measurable are upper radical classes defined by classes of slender objects. Using properties of slender abelian groups and a version of slenderness for rings, we get some examples of radical classes of associative rings which are closed under (non-measurable) products.

There is also a notion of slenderness for modules, first considered by Allouch [2],[3]. Some radical implications of this will also be described.

Two other closure properties for radical classes will be discussed: closure 
under surjective inverse limits (i.e. limits of inverse systems whose homomorphisms are surjective) and closure under essential extensions. Closure under surjective inverse limits is stronger than product closure. Essential closure is pertinent because of a result of Loi [57]: every essentially closed radical class of associative rings is a variety. Veldsman [77] proved a related result for nearrings and in Section 9 we prove the exact analogue of Loi's result for groups. In view of the fact that for modules essential closure is quite innocuous, and has no connection with product closure, these results seem a little mysterious and the relationship between essential closure and product closure deserves further exploration.

Notation and terminology are mostly standard. For anything unexplained see [38] for radical theory, [23] for abelian groups and [52] for set theory and cardinal numbers. Here are a few things to note.

The lower radical class defined by a class $\mathcal{M}$ (the smallest radical class containing $\mathcal{M})$ is denoted by $L(\mathcal{M})$ and the upper radical class defined by $\mathcal{M}$ (the largest radical class for which everything in $\mathcal{M}$ is semi-simple) is denoted by $U(\mathcal{M})$. The latter may not exist in some settings, e.g. non-associative rings, but it always does for associative rings, groups, abelian groups and modules. We write $L(A), U(A)$ rather than $L(\{A\}), U(\{A\})$ in the case of a class with a unique member. The Jacobson radical class is called $\mathcal{\partial}$.

We use "direct product" and "product" interchangeably. We denote the product of $\left\{A_{\lambda}: \lambda \in \Lambda\right\}$ by $\prod_{\lambda \in \Lambda} A_{\lambda}$ and regardless of context call the subobject $\bigoplus_{\lambda \in \Lambda} A_{\lambda}$ consisting of all elements with finite support the direct sum. If $|\Lambda|=\aleph_{0}$ (resp. $|\Lambda|$ is non-measurable, resp. $|\Lambda|=\mathfrak{a}$ for some cardinal number $\mathfrak{a}$ ) we call $\prod_{\lambda \in \Lambda} A_{\lambda}$ a countable product (resp. a non-measurable product, resp. an $\mathfrak{a}$-product). If all the $A_{\lambda}$ are equal to some $A$, we write $A^{\Lambda}, A^{(\Lambda)}$ for $\prod_{\lambda \in \Lambda} A_{\lambda}, \bigoplus_{\lambda \in \Lambda} A_{\lambda}$. In particular when $\Lambda$ is countably infinite, we write $A^{\omega}, A^{(\omega)}$ ( $\omega$ being the set of finite ordinals).

\section{Generalities}

If a radical class $\mathcal{R}$ contains $A_{\lambda}$ for all $\lambda \in \Lambda$ then $\bigoplus_{\lambda \in \Lambda} A_{\lambda} \in \mathcal{R}$ so by closure under extensions we have

Proposition 2.1. A radical class $\mathcal{R}$ is closed under products (a-products) if and only if $\prod_{\lambda \in \Lambda} A_{\lambda} / \bigoplus_{\lambda \in \Lambda} A_{\lambda} \in \mathcal{R}$ whenever $A_{\lambda} \in \mathcal{R}$ for all $\lambda \in \Lambda$ (and $|\lambda| \leq \mathfrak{a})$.

Thus the nature of structures $\prod / \bigoplus$ has relevance to our problem. $\mathrm{Hu}$ lanicki's memorable result [48] that for all abelian groups $A_{1}, A_{2}, \ldots, A_{n}, \ldots$ the group $\prod A_{n} / \bigoplus A_{n}$ is algebraically compact is used to prove that every radical class of abelian groups which is determined as a lower radical class 
by torsion-free groups is closed under countable products. See Section 3 for further information. The following result is probably well known.

Proposition 2.2. A radical class is closed under direct products if and only if it is closed under direct powers.

Proof. If a radical class $\mathcal{R}$ is closed under direct powers and $A_{\lambda} \in \mathcal{R}$ for all $\lambda \in \Lambda$ then $\bigoplus_{\lambda \in \Lambda} A_{\lambda} \in \mathcal{R}$, so $\left(\bigoplus_{\lambda \in \Lambda} A_{\lambda}\right)^{\Lambda} \in \mathcal{R}$. For each $\mu \in \Lambda$ the projection $\pi_{\mu}: \bigoplus_{\lambda \in \Lambda} A_{\lambda} \rightarrow A_{\mu}$ is a surjective homomorphism and we get a surjective homomorphism $\left(\bigoplus_{\lambda \in \Lambda} A_{\lambda}\right)^{\Lambda} \rightarrow \prod_{\lambda \in \Lambda} A_{\lambda}$ from the correspondence $\left(\left(a_{\lambda}^{\mu}\right)\right)_{\mu} \mapsto\left(a_{\mu}^{\mu}\right)_{\mu}$

The following quite general result provides a sufficient condition for failure of product closedness. It plays a part in the papers of Olszewski [64] and Phillips [67].

Proposition 2.3. Let

$$
\mathrm{e}_{1} \subsetneq \mathrm{e}_{2} \subsetneq \ldots \subsetneq \mathrm{e}_{\alpha} \subsetneq \ldots
$$

be an infinite chain of homomorphically closed classes labelled by ordinals so that $\mathrm{C}_{\alpha} \subsetneq \mathrm{C}_{\gamma}$ if and only if $\alpha<\gamma$. Further suppose that for each limit ordinal $\beta$ we have $\mathcal{C}_{\beta}=\bigcup_{\alpha<\beta} \mathcal{C}_{\alpha}$. If there is a limit ordinal $\lambda$ such that $\mathcal{C}_{\lambda}$ is the union of all the classes and $\lambda$ is the smallest such ordinal then $\mathcal{C}_{\lambda}$ is not closed under products. This applies in particular when $\mathcal{C}_{\lambda}$ is a radical class.

Proof. For each $\alpha<\lambda$ let $A_{\alpha+1}$ be in $\mathcal{C}_{\alpha+1} \backslash \mathcal{C}_{\alpha}$. Suppose $\prod_{\alpha<\lambda} A_{\alpha+1} \in \mathcal{C}_{\lambda}$. Then $\prod_{\alpha<\lambda} A_{\alpha+1} \in \mathcal{C}_{\delta}$ for some $\delta<\lambda$. But $\mathcal{C}_{\delta}$ is homomorphically closed so $A_{\delta+1} \in \mathcal{C}_{\delta}$ and we have a contradiction.

Corollary 2.4. Let $\mathcal{M}$ be a homomorphically closed class such that the Kurosh construction of $L(\mathcal{M})$ terminates at a limit ordinal. Then $L(\mathcal{M})$ is not closed under direct products.

Proof. For ordinals $\alpha$ let $\mathcal{M}_{\alpha}$ be the usual intermediate classes in the Kurosh construction. (with $\mathcal{M}_{1}=\mathcal{M}$ ). Then the conditions of $\mathbf{2 . 3}$ are met.

Corollary 2.5. If $\mathcal{R}$ is a product-closed radical class of associative rings, alternative rings, groups, Jordan algebras over a field of characteristic $\neq 2$ or Hausdorff topological associative rings, then any Kurosh construction of $\mathcal{R}$ as a lower radical class terminates in finitely many steps. 
This is because in all the indicated cases the Kurosh construction stops either after finitely many steps or at the step corresponding to the first infinite ordinal. See [33], pp. 38,48,30,55,57. Only in the case of associative rings is it known that infinitely steps may be necessary (Heinicke [45]; see also [38], p.64).

It should be noted that no conclusion can be reached about a radical class simply because it has no infinite construction as a lower radical class. Note also that if a class is product-closed its lower radical class need not be.

We end this section with a table showing how some of the standard associative ring radicals behave with respect to products.

\begin{tabular}{|l|l|}
\hline & Product closed? \\
\hline Prime & No \\
\hline Nil & No \\
\hline Jacobson & Yes \\
\hline Brown-McCoy & No (Olszewski[64]) \\
\hline Generalized nil & No \\
\hline Regular & Yes \\
\hline Hereditarily idempotent & Yes(?)(Jeon, Kim, Lee [53]) \\
\hline Idempotent & No \\
\hline
\end{tabular}

(It is claimed in [53] that products of hereditarily idempotent rings are hereditarily idempotent, but this is unclear. A product of infinitely many commutative nilpotent rings where there is no bound on the indices is not nil; this takes care of the prime, nil and generalized nil radicals. For each $n \in \mathbb{Z}^{+}$ there is an idempotent ring $R_{n}$ containing an element $r_{n}$ which is a sum of $n$ products but no fewer. (For an example, see [9], Lemma 31.) In $\prod R_{n},\left(r_{n}\right)$ is not a sum of products.)

\section{Abelian Groups}

The first result dealing with product closure for radical classes of abelian groups (and probably for radical classes in any setting) makes essential use of Hulanicki's theorem [48] that a countable product modulo the sum is algebraically compact (see Section 1).

Example 3.1. A radical class $\mathcal{R}$ of abelian groups is closed under countable direct products if and only if $\mathcal{R}=L(\mathcal{F})$ for some class $\mathcal{F}$ of torsion-free groups.

[25], Theorem 7.2. 
Ivanov [50] generalized this by showing that for every radical class $\mathcal{R}$ of abelian groups the class of torsion-free groups in $\mathcal{R}$ is closed under countable direct products.

For a set $S$ of primes an abelian group $G$ is $S$-divisible if $G=p G$ for every $p \in S$. The class $\mathcal{D}_{S}$ of $S$-divisible abelian groups is $L(\mathbb{Q}(S))$, where $\mathbb{Q}(S)$ is the group of rational numbers whose denominators (in reduced form) are products of powers of primes in $S$.

Example 3.2. $\mathcal{D}_{S}$ is a product closed radical class of abelian groups.

Are there radical classes which are closed under countable, but not arbitrary products? From the proof of Theorem 2.4(2) of Dugas and Göbel [19] we get the following.

Example 3.3. For every infinite regular cardinal $\mathfrak{k}$ (e.g. $\mathfrak{k}=\aleph_{0}$ ) there is a class of torsion-free abelian groups whose lower radical class is not closed under products of $\mathfrak{k}^{+}$groups.

This example is based on rigid systems of abelian groups. A rigid system is a set $\mathcal{X}$ of abelian groups such that $\operatorname{Hom}(A, B)=0$ for all distinct $A, B \in \mathcal{X}$ and $\operatorname{Hom}(A, A)$ is torsion-free of rank 1 for each $A \in X$. There is, for every regular cardinal $\mathfrak{k}$, a rigid system $\mathcal{X}$ of torsion-free cotorsion-free abelian groups with $|X|=\mathfrak{k}^{+}[74]$. If

$$
G=\prod\{A: A \in X\} / \prod^{<\mathfrak{k}^{+}}\{A: A \in X\},
$$

where $\prod^{<\mathfrak{k}^{+}}$denotes the subgroup of elements with support of cardinality $<\mathfrak{k}^{+}$, then $G \notin L(X)$. A critical role in the proof of this is played by the following result.

Lemma 3.4. (Wald-Łoś Lemma.) Let $\left\{G_{\lambda}: \lambda \in \Lambda\right\}$ be a set of abelian groups with $|\Lambda|$ regular, $\Phi$ a $|\Lambda|$-complete filter on $\Lambda$, i.e. a filter containing all intersections of $<|\Lambda|$ of its members. Then every injective homomorphism $f: A \rightarrow \prod_{\lambda \in \Lambda} G_{\lambda} / \Phi$ with $|A|<|\Lambda|$ lifts to an injective homomorphism $\hat{f}:$ $A \rightarrow \prod_{\lambda \in \Lambda} G_{\lambda}$ with $f=\pi \hat{f}, \pi$ being the natural homomorphism $\prod_{\lambda \in \Lambda} G_{\lambda} \rightarrow$ $\prod_{\lambda \in \Lambda} G_{\lambda} / \Phi$.

A special case, using a rigid system of torsion-free groups of rank 1, is described in [33], p.165. The radical class in that case is closed under countable direct products but not under direct products of $\aleph_{1}$ groups.

For a prime $p$ let $\mathbb{Q}(p)=\left\{\frac{m}{p^{n}}: m, n \in \mathbb{Z}\right\}$. Our next example was obtained by a totally different argument.

Example 3.5. The lower radical class defined by $\{\mathbb{Q}(p), \mathbb{Q}(q)\}$, for distinct primes $p, q$, is not closed under uncountable direct products ([35],Theorem 2.6). 
By Example 3.2, $L(\mathbb{Q}(p))=\mathcal{D}_{p}$, the class of $p$-divisible groups, which is product-closed. Thus $L(\{\mathbb{Q}(p), \mathbb{Q}(q)\}$ is a minimal counterexample for showing that countable product closure does not imply product closure.

A torsion-free abelian group $G$ is slender if for every homomorphism $f$ : $\mathbb{Z}^{\omega} \rightarrow G$ we have $f(\mathbb{Z})=0$ for almost all copies of $\mathbb{Z}$. This notion is due to Łoś; for an account see [23], Vol. II, pp. 158-166. We'll see that upper radical classes defined by classes of slender groups are closed under large direct products but it is not known whether they are closed under arbitrary ones. To make our meaning clear we need the notion of a measurable cardinal.

A cardinal number $\mathfrak{a}$ is measurable if a set $E$ with cardinality $\mathfrak{a}$ supports a countably additive measure $\mu$ which takes only the values 0 and 1 such that $\mu(E)=1$ and $\mu(\{x\})=0$ for each $x \in E$. Then there is an $\mathfrak{a}$-additive measure on $E$.

We also need the following theorem.

Theorem 3.6. (Łoś; see [23], Vol.II, p.161.) Let $G$ be a slender abelian group, $A_{\lambda}, \lambda \in \Lambda$ abelian groups, where $|\Lambda|$ is not measurable. If $f: \prod_{\lambda \in \Lambda} A_{\lambda} \rightarrow G$ is a homomorphism such that $\bigoplus_{\lambda \in \Lambda} A_{\lambda} \subseteq \operatorname{Ker}(f)$, then $f=0$.

The proof in [23] is for torsion-free $A_{\lambda}$, but this is not a real limitation and it essentially deals with the restriction of $f$ to subgroups which are direct products of (infinite) cyclic groups. For the sake of completeness, and to indicate the style of argument, let us assume the result for the products of infinite cyclic groups and use this to prove the general version.

Let $a=\left(a_{\lambda}\right)_{\Lambda}$ be in $\prod_{\lambda \in \Lambda} A_{\lambda}$. Then $a \in \prod_{\lambda \in \Lambda}\left\langle a_{\lambda}\right\rangle \subseteq \prod_{\lambda \in \Lambda} A_{\lambda}$. Let $\left\langle e_{\lambda}\right\rangle$ be an infinite cyclic group for each $\lambda \in \Lambda$. Then there is a homomorphism $g: \prod_{\lambda \in \Lambda}\left\langle e_{\lambda}\right\rangle \rightarrow \prod_{\lambda \in \Lambda}\left\langle a_{\lambda}\right\rangle$ with $g\left(\left(e_{\lambda}\right)_{\Lambda}\right)=\left(a_{\lambda}\right)_{\Lambda}=a$ and $g\left(e_{\lambda}\right)=a_{\lambda}$ for every $\lambda$. We then have $f g\left(\left\langle e_{\lambda}\right)\right\rangle \subseteq f\left(A_{\lambda}\right)=0$ for all $\lambda$ so (by what we are assuming) $f g$ is the zero map. Hence $f(a)=f g\left(\left(e_{\lambda}\right)_{\Lambda}\right)=0$. Since $a$ is arbitrary, we conclude that $f=0$ as required.

If $S$ is a slender group and $\operatorname{Hom}\left(G_{\lambda}, S\right)=0$ for each $\lambda \in \Lambda$, where $|\Lambda|$ is non-measurable, then every homomorphism $\prod_{\lambda \in \Lambda} G_{\lambda} \rightarrow G$ takes each $G_{\lambda}$ to 0 and so must be the zero map.

The following is now clear.

Example 3.7. The upper radical class defined by a class of slender groups is closed under direct products for which the cardinality of the set of factors is not measurable.

It is not known whether or not there are any measurable cardinals, but if there are they are very large. Again we refer to [52] for information. 
A useful characterization of slender abelian groups is due to Nunke [62]: an abelian group is slender if and only if it is torsion-free and has no subgroup isomorphic to $\mathbb{Q}, \mathbb{Z}^{\omega}$ or the $p$-adic integers for any prime $p$. This subsumes an earlier result of Saşiada: a countable torsion-free abelian group is slender if and only if it is reduced.

Another possible source of product-closed radical classes of abelian groups is a construction of Nunke [63] which we now describe.

Let

$$
0 \rightarrow \mathbb{Z} \rightarrow A \rightarrow B \rightarrow 0
$$

be a short exact sequence of abelian groups. For each abelian group $G$ there is an induced homomorphism

$$
\operatorname{Hom}(A, G) \rightarrow \operatorname{Hom}(\mathbb{Z}, G) \cong G .
$$

If we let $r(G)$ be the image of this homomorphism, we thereby define a subfunctor $r$ of the identity which commutes with arbitrary direct products. Sometimes this $r$ is a (Kurosh-Amitsur) radical (what abelian category people call an idempotent radical); then the corresponding radical class is product closed. For instance (with the notation above) for the sequence

$$
0 \rightarrow \mathbb{Z} \rightarrow \mathbb{Q}(S) \rightarrow \bigoplus_{p \in S} \mathbb{Z}\left(p^{\infty}\right) \rightarrow 0
$$

we have

$$
r(G)=\operatorname{Im}\left(\operatorname{Hom}(\mathbb{Q}(S), G) \rightarrow \operatorname{Hom}(\mathbb{Z}, G) \cong G=\mathcal{D}_{S}(G) .\right.
$$

We don't actually have any other examples, just as we have no other examples besides the $\mathcal{D}_{S}$ of non-trivial radical classes closed under direct products. The problem is to find middle terms for short exact sequences so that the resulting functor is a radical. There is some information in [31], but it is not very definitive. It is shown, however that the $p$-adic integers with the 1-preserving embeddding of $\mathbb{Z}$ can't be used in this way. Note that this is not to say that the lower radical class defined by the $p$-adic integers is not product closed: this is still an open question.

Modern abelian group theory has lots of interactions with set theory; in particular some accepted results depend on which axioms are added to ZFC. A famous example is Shelah's solution to the Whitehead Problem: for which $G$ is $\operatorname{Ext}(G, \mathbb{Z})=0$ ? With Gödel's Axiom of Constructibility $G$ must be free, but with Martin's Axiom and $2^{\aleph_{0}}>\aleph_{1}$ it need not be [74]. We now note a connection between radical theory and set theory.

We say that a radical class $\mathcal{R}$ of abelian groups satisfies the Cardinality Condition if there is a cardinal number $\mathfrak{a}$ such that for every abelian group $G$ we have 


$$
\mathcal{R}(G)=\sum\{H: H \text { is a subgroup of } G, H \in \mathcal{R} \text { and }|H|<\mathfrak{a}\} .
$$

(The notion has been formulated a bit differently for subfunctors of the identity in general, but for Kurosh-Amitsur radicals the definition coincides with what we have given. There are cardinality conditions in other settings too: for rings we have, e.g., a Subring Cardinality Condition and an Ideal Cardinality Condition, cf. [34]; it follows from a result of Casacuberta et al. [13] that verbal radicals of groups satisfy the Cardinality Condition for subgroups.)

A subfunctor of the identity (of abelian groups) is definable from a short exact sequence by Nunke's method if and only if it contains the divisible subfunctor, commutes with direct products and satisfies the Cardinality Condition ([63], Theorem 2.2). Since every radical class of abelian groups which contains non-torsion groups must contain all divisible groups [26] it follows that a product closed radical class of abelian groups is definable by a sequence if and only if it satisfies the Cardinality Condition. (See also [31], Theorem 2.1 for this special case.)

Now Eda [21] has shown that under the Vopěnka Principle (see [52]) all subfunctors of the identity satisfy the cardinality condition and hence if they commute with products are defined by short exact sequences. Thus under the Vopěnka Principle, all product closed radical classes are definable by sequences and so the elusive "middle terms" are neither more nor less hard to find than the product closed radical classes of abelian groups.

We end this section by mentioning an idea from abelian groups which is also worth considering in other contexts.

Corner and Göbel [14] define the norm of a subfunctor of the identity. Specializing this to Kurosh-Amitsur radicals and transferring it to radical classes, we say the norm of a radical class $\mathcal{R}$ is the smallest cardinal number $\mathfrak{m}$ such that $\mathcal{R}$ is not closed under direct products of $\mathfrak{m}$ factors, or $\infty$ if there is no such cardinal. Here are some examples for abelian groups.

$\aleph_{0}$ : the class of torsion groups.

$\aleph_{1}: L(\{\mathbb{Q}(p), \mathbb{Q}(q)\})$ for distinct primes $p, q[35]$; the Chase radical (the upper radical class defined by the class of $\aleph_{1}$-free abelian groups. This was proved by Eda [20] with the Continuum Hypothesis and by Pokutta and Strüngman [68] with ZFC.

$\infty: \mathcal{D}_{S}$ for a set $S$ of primes.

These, via A-radicals, provide examples for rings, but one might hope for something more interesting there. 


\section{Abelian $\ell$-groups}

As each class of torsion-free abelian groups defines a lower radical class (in abelian groups) closed under countable products, it seems natural to ask whether there is a variety of multioperator groups in which the underlying groups are torsion-free abelian and in which all radical clases are closed under countable products (cf. also the result of Ivanov [50] cited above). An obvious candidate is the class of abelian $\ell$-groups, and some encouragement may be obtained from a result of Hager and Madden [43]: in torsion-free abelian groups the classes $\mathcal{D}_{S}$ of $S$-divisible groups are precisely the non-trivial classes closed under products, homomorphic images and pure subgroups, while the non-trivial classes of abelian $\ell$-groups which are closed under products, homomorphic images and $\ell$-subgroups are the classes $\mathcal{D}_{S}^{\ell}=\left\{(G, \leq): G \in \mathcal{D}_{S}\right\}$. The $\mathcal{D}_{S}$ are the non-trivial radical classes closed under products and pure subgroups (see [24]) so the $\mathcal{D}_{S}^{\ell}$ are the radical classes of abelian $\ell$-groups which are closed under products and $\ell$-subgroups.

We shall show, however, that radical classes of abelian $\ell$-groups need not be closed under countable products. Our counterexample will be the lower radical class $L(\mathbb{Q})$ defined by $\mathbb{Q}$ with its standard order. (In abelian groups the lower radical defined by $\mathbb{Q}$ is the class of divisible groups.)

Example 4.1. In abelian $\ell$-groups, $\mathbb{Q}^{\omega} / \mathbb{Q}^{(\omega)}$ is $L(\mathbb{Q})$-semi-simple, so $L(\mathbb{Q})$ is not closed under countable products.

In $\mathbb{Q}^{\omega} / \mathbb{Q}^{(\omega)}$ let $\left(a_{n}\right)+\mathbb{Q}^{(\omega)}=\left[a_{n}\right]$. Then $\left[a_{n}\right] \vee\left[b_{n}\right]=\left(a_{n}\right) \vee\left(b_{n}\right)+\mathbb{Q}^{(\omega)}=$ $\left[a_{n} \vee b_{n}\right]$, so $\left[a_{n}\right]<\left[b_{n}\right]$ if and only if $\left[a_{n}\right] \neq\left[b_{n}\right]$ and $\left[a_{n} \vee b_{n}\right]=\left[a_{n}\right] \vee\left[b_{n}\right]=\left[b_{n}\right]$, i.e. $a_{n} \vee b_{n}=b_{n}$ for almost all $n$ and $a_{n} \neq b_{n}$ for infinitely many $n$.

Let $\left[a_{n}\right]$ be $<\left[b_{n}\right]$ and let $S=\left\{n: a_{n} \vee b_{n}=b_{n}\right\}$ and $T=\left\{n: a_{n} \neq b_{n}\right\}$. Then $S \cap T=\left\{n: a_{n}<b_{n}\right\}$ is infinite. For each $E \subseteq S \cap T$ let $\left(x_{n}^{E}\right) \in \omega$ be defined by

$$
x_{n}^{E}= \begin{cases}\frac{a_{n}+b_{n}}{2} & \text { if } n \in E \\ a_{n} & \text { if } n \in S \backslash E . \\ 0 & \text { if } n \notin S .\end{cases}
$$

Then $a_{n}=x_{n}^{E} \leq b_{n}$ for all $n \in S \backslash E$ and $a_{n}<x_{n}^{E}<b_{n}$ for all $n \in E$. Hence $\left[a_{n}\right] \leq\left[x_{n}^{E}\right] \leq\left[b_{n}\right]$ and if $E$ is infinite then $\left[a_{n}\right]<\left[x_{n}^{E}\right]<\left[b_{n}\right]$. Since $S \cap T$ is infinite, it has $2^{\aleph_{0}}$ infinite subsets, and if $E$ and $F$ are two such then $\left[x_{n}^{E}\right]=\left[x_{n}^{F}\right]$ if and only if $x_{n}^{E}=x_{n}^{F}$ for almost all $n$. Now 


$$
x_{n}^{E}-x_{n}^{F}= \begin{cases}0 & \text { if } n \in E \cap F \\ \frac{a_{n}+b_{n}}{2}-a_{n}=\frac{b_{n}-a_{n}}{2} & \text { if } n \in E \backslash F \\ a_{n}-\frac{a_{n}+b_{n}}{2}=\frac{a_{n}-b_{n}}{2} & \text { if } n \in F \backslash E \\ 0 & \text { if } n \in(S \backslash E) \cap(S \backslash F)=S \backslash(E \cup F) \\ 0 & \text { if } n \notin S\end{cases}
$$

Since $E, F \subseteq S \cap T$ we have $a_{n} \neq b_{n}$ for all $n \in E$ or $F$, it follows that $x_{n}^{E}=x_{n}^{F}$ for almost all $n$ if and only if $E \backslash F$ and $F \backslash E$ are finite, i.e. $E \triangle F$ is finite.

Let $\sigma$ be the relation on $S$ defined as follows.

$$
E \sigma F \text { if and only if } E \triangle F \text { is finite. }
$$

(Here $\triangle$ denotes the symmetric difference.) Clearly $\sigma$ is reflexive and symmetric. If $E \sigma F$ and $F \sigma G$, then

$$
E \triangle G=E \triangle(F \triangle F) \triangle G=(E \triangle F) \triangle(F \triangle G)
$$

and this is finite. Thus $\sigma$ is an equivalence relation.

The equivalence class $F \sigma$ of $F$ is

$\{E \subseteq S \cap T: E \backslash F$ and $F \backslash E$ are finite $\}$

$=\{E \subseteq S \cap T: E=(F \backslash A) \cup B$ for finite sets $A, B$ with $A \subseteq F$ and $B \cap F=\emptyset\}$.

As $\mathbb{Z}$ has only countably many finite subsets, each $E \sigma$ is countable. Hence there are uncountably many distinct $\left[x_{n}^{F}\right]$, all of which are strictly between $\left[a_{n}\right]$ and $\left[b_{n}\right]$.

We conclude that all intervals in $\mathbb{Q}^{\omega} / \mathbb{Q}^{(\omega)}$ are uncountable. But then $\mathbb{Q}^{\omega} / \mathbb{Q}^{(\omega)}$ has no non-zero countable convex subgroups and in particular no convex subgroup isomorphic to $\mathbb{Q}$ whence $\mathbb{Q}^{\omega} / \mathbb{Q}^{(\omega)}$ is $L(\mathbb{Q})$-semi-simple.

\section{Groups}

A nice result of Shmel'kin [75] tells us that all semi-simple classes of groups are closed under free products. Free products are coproducts, so it is another indication of the lack of duality in radical theory (cf. [37]) that there are radical classes of groups which are not product closed.

We have a characterization, due to Göbel [41], of strict radical classes of groups which are closed under countable direct products. Interestingly, this result was obtained not from a radical-theoretic investigation but from the study of $\mathcal{E}$ - perfect groups for a class $\mathcal{E}$ : those groups without nontrivial images in $\mathcal{E}$. (When $\mathcal{E}$ is the class of abelian groups we get the more 
established notion of perfect groups.) If $\mathcal{E}$ is a strongly hereditary class, the $\mathcal{E}$-perfect groups form a strict radical class, but in general they need not form a radical class: for example if $p$ is a prime, then the cyclic group $\mathbb{Z}(p)$ is $\left\{\mathbb{Z}\left(p^{\infty}\right)\right\}$-perfect, but any radical class containing $\mathbb{Z}(p)$ also contains $\mathbb{Z}\left(p^{\infty}\right)$.

The characterization involves the non-abelian version of slender groups and a related class, similarly connected with cotorsion-free abelian groups.

A group $G$ is slender if it is torsion-free and every homomorphism $\mathbb{Z}^{\omega} \rightarrow G$ takes almost all components to the identity of $G$.

Proposition 5.1. The following conditions are equivalent.

(i) $G$ is slender.

(ii) If $\left\{F_{\lambda}: \lambda \in \Lambda\right\}$ is a set of groups and $|\Lambda|$ is not measurable, then for every homomorphism $f: \prod_{\lambda \in \Lambda} F_{\lambda} \rightarrow G$,

(a) $f$ takes almost all $F_{\lambda}$ to the identity of $G$;

(b) $f$ is the trivial map if (and only if) it takes every $F_{\lambda}$ to the identity of $G$.

(iii) All abelian subgroups of $G$ are slender.

([41], Lemma 3.6).

Thus by Nunke's result [62] a torsion-free group is slender if and only if it has no subgroup isomorphic to $\mathbb{Q}, \mathbb{Z}^{\omega}$ or any $p$-adic integers.

A group $G$ is stout if it is torsion-free and there is a group $Y$ with $|Y|>2$ such that every homomorphism $f:(Y * Y)^{\omega} \rightarrow G$ is trivial; here $Y * Y$ is the free product.

Theorem 5.2. The following conditions are equivalent for a torsion-free group $G$.

(i) All abelian subgroups of $G$ are stout.

(ii) All abelian subgroups of $G$ are cotorsion-free.

(iii) If $\left\{F_{\lambda}: \lambda \in \Lambda\right\}$ is a set of groups with $|\Lambda|=\aleph_{0}$ and all homomorphisms $F_{\lambda} \rightarrow G$ are trivial, for all $\lambda$, then all homomorphisms $\prod_{\lambda \in \Lambda} F_{\lambda} \rightarrow G$ are trivial.

[41], Theorem 4.1.

The stout groups are torsion-free and have no subgroups isomorphic to $\mathbb{Q}$ or any $p$-adic integers. They differ from the slender groups in allowing subgroups isomorphic to $\mathbb{Z}^{\omega}$. Clearly slender groups are stout (which makes the terminology rather unsuggestive).

The upper radical class $U(\mathcal{E})$ defined by a strongly hereditary class of groups is strict, i.e. in any group the radical contains all radical subgroups. Consequently $Y * Y \in U(\mathcal{E})$ for every $Y \in U(\mathcal{E})$. If $U(\mathcal{E})$ is closed under countable products, then there can be no non-trivial homomorphisms from $(Y * Y)^{\omega}$ to any group in $\mathcal{E}$ (as the latter is strongly hereditary). Choosing $|Y|>2$ (which 
clearly is not difficult) we see that $\mathcal{E}$ consists of stout groups (unless, of course, it consists of all groups).

It turns out that the converse is also true. An important step in the proof is showing that if $|Y|>2$ then $(Y * Y)^{\omega}$ can be mapped onto every finite cyclic group (see [40], Satz 4.2) which in turn relies on a theorem of Rhemtulla [70] concerning bounded expressibility in free products. All the groups of $p$-adic integers are also homomorphic images of $(Y * Y)^{\omega}$ for $Y$ as above (see [41], Lemma 3.1). Here is the result.

Example 5.3. A strict radical class $\mathcal{R}$ of groups is closed under countable direct products if and only if $\mathcal{R}=\{1\}$ or $\mathcal{R}$ is the upper radical class defined by a class of stout groups.

(Cf. [41], Theorem 5.1.)

Recall that a (necessarily strict) radical class of abelian groups is closed under countable direct products if and only if it is the lower radical class defined by a class of torsion-free groups. It is interesting to compare this result with the one just stated. A radical class of abelian groups which is the lower radical class defined by torsion-free groups need not be determined by torsion-free groups (and a fortiori not by cotorsion-free ones) as an upper radical class. For instance $\mathcal{D}_{p}=L(\mathbb{Q}(p))$ and $\mathcal{D}_{p}(\mathbb{Z}(p))=0$ but $\mathbb{Z}(p)$ is not in any semi-simple class defined by torsion-free groups. On the other hand, "stout" means "cotorsion-free" for abelian groups and by Hulanicki's theorem we have the following.

Example 5.4. In abelian groups, the upper radical class defined by a class of cotorsion-free groups is closed under countable direct products.

Whether or not a radical class (strict or otherwise) of groups need be closed under countable products if it is determined as a lower radical class by torsion-free groups appears to be unknown.

Example 5.5. The class of groups without subgroups of finite index is a strict radical class which is not closed under countable products.

As this class is the upper radical class defined by all finite groups, this follows from Example 5.3. The result was recently proved by Gismatullin and Muranov [39] who also used the theorem of Rhemtulla referred to above.

Let $\mathcal{S}$ be a class of slender groups. If $G_{\lambda} \in U(\mathcal{S})$ for all $\lambda \in \Lambda$ and $|\Lambda|$ is non-measurable, let $f: \prod_{\lambda \in \Lambda} G_{\lambda} \rightarrow H \in \mathcal{S}$ be a homomorphism with accessible image. Then for each $\lambda$ the restriction of $f$ to $G_{\lambda}$ has accessible image and so $\left(G_{\lambda}\right.$ being in $\left.U(\mathcal{S})\right)$ is trivial. But then so is $f$. We conclude that $\prod_{\lambda \in \Lambda} G_{\lambda} \in U(\mathcal{S})$. Thus we have 
Theorem 5.6. The upper radical class defined by every class of slender groups is closed under non-measurable products.

We now present a few illustrations.

Example 5.7. Let $\mathcal{F}$ denote the class of free groups and the one element group. This is strongly hereditary and its only abelian members are cyclic. Hence $\mathcal{F}$ consists of slender groups, so $U(\mathcal{F})$ is closed under non-measurable products.

Example 5.8. For a cardinal number $\mathfrak{a}$ let $\mathcal{F}_{\mathfrak{a}}$ (resp. $\overline{\mathcal{F}_{\mathfrak{a}}}$ ) be the class of free groups with rank $>\mathfrak{a}($ resp. $\geq \mathfrak{a})$. If $N \triangleleft F \in \mathcal{F}_{\mathfrak{a}}$ or $\overline{\mathcal{F}_{\mathfrak{a}}}$ then $|N|=1$ or $N$ is free, and in the latter case $N$ has rank at least as great as that of $F$. (See [75].) Thus $\mathcal{F}_{\mathfrak{a}}$ and $\overline{\mathcal{F}_{\mathfrak{a}}}$ are hereditary and both $U\left(\mathcal{F}_{\mathfrak{a}}\right)$ and $U\left(\overline{\mathcal{F}}_{\mathfrak{a}}\right)$ are closed under non-measurable products.

Example 5.9. Each free group can be embedded in a group in which all elements apart from the identity are conjugates, by a construction of Higman, Neumann and Neumann [47]. The containing group is clearly simple. Moreover, the subgroups of the containing group are slender so their upper radical class is closed under non-measurable direct products.

This example is discussed in [41], Theorem 5.2.

Example 5.10. Let $S$ be a slender simple group (for example the group containing the free group in the previous example). Then $U(S)$ is closed under non-measurable products.

Note that the radical classes in Examples 5.8 and 5.10 are not strict.

\section{Associative rings and slenderness}

By using $A$-radicals we can obtain examples of radical classes of associative rings satisfying various properties - product closure, closure under countable but not arbitrary products and so on - from radical classes of abelian groups with these properties. In particular we have.

Example 6.1. If $\mathcal{R}$ is a radical class of abelian groups which is the upper radical class defined by a class of slender groups, then the corresponding $A$ radical class of associative rings is closed under non-measurable products.

We can do a bit better in this case though.

Proposition 6.2. Let $\mathcal{C}$ be a class of associative rings whose additive groups are slender. Then $U(\mathcal{C})$ is closed under non-measurable products. 
Proof. Let $A$ be in $\mathcal{C}, R_{\lambda} \in U(\mathcal{C})$ for all $\lambda \in \Lambda$ where $|\Lambda|$ is non-measurable. Let $f: \prod_{\lambda \in \Lambda} R_{\Lambda} \rightarrow A$ be a homomorphism with accessible image. Then $\bigoplus_{\lambda \in \Lambda} R_{\lambda} \in U(\mathrm{C})$ and $\left.f\right|_{\oplus_{\lambda \in \Lambda} R_{\lambda}}$ has accessible image and so is zero. On purely additive grounds we conclude that $f=0$, whence it follows that $\prod_{\lambda \in \Lambda} R_{\lambda} \in U(\mathrm{C})$.

Example 6.3. $U(\mathbb{Z})$ is a strict special radical class closed under non-measurable products.

Example 6.4. $U\left(\mathbb{Z}^{0}\right)$ is closed under non-measurable products and consists of idempotent rings. Here $\mathbb{Z}^{0}$ is the zeroring on $\mathbb{Z}$.

Example 6.5. Let $2 \mathbb{Q}_{(2)}=\left\{\frac{m}{n}: m, n \in \mathbb{Z} \& 2 \mid m \& 2 \nmid n\right\}$. Then $U\left(2 \mathbb{Q}_{(2)}\right)$ is special and closed under non-measurable products. Hence $U\left(2 \mathbb{Q}_{(2)}\right) \cap \mathcal{J}$ is special, closed under non-measurable products and properly contained in $\mathcal{J}$.

In [36] we have presented a version of slenderness for rings. A torsionfree ring $R$ is slender if for every ring homomorphisn $f: \mathbb{Z}^{\omega} \rightarrow R$ there exist $i_{1}, i_{2}, \ldots, i_{n}$ such that $f\left(\left(a_{i}\right)\right)=\sum_{j=1}^{n} f\left(a_{i_{j}} e_{i_{j}}\right)$ for all $\left(a_{i}\right) \in \mathbb{Z}^{\omega}$, where $e_{i} \in \mathbb{Z}^{\omega}$ is the identity of the $i$ th copy of $\mathbb{Z}$.

The involvement of the ring of integers makes this version of slenderness less applicable to radical theory than the original or the group version. For this and other reasons it might be interesting to study another version in which the role of $\mathbb{Z}$ is taken by a free ring. Nevertheless we do have one result based on the given notion of slenderness for rings.

Proposition 6.6. If $S$ is a slender simple associative ring with identity and characteristic 0, then $U(S)$ is closed under non-measurable products.

Proof. Let $R_{\lambda}$ be in $U(S)$ for each $\lambda \in \Lambda$ where $|\Lambda|$ is non-measurable. Let $R_{\lambda}^{1}=R$ if $R$ has an identity and the standard unital extension otherwise. Then $\mathbb{Z} \in U(S)$ so each $R_{\lambda}^{1}$ is in $U(S)$. Suppose there is a surjective ring homomorphism $f: \prod_{\lambda \in \Lambda} R_{\lambda}^{1} \rightarrow S$. Then each $R_{\lambda}^{1}$ is mapped to an ideal of $S$ and hence to 0 . Thus $f\left(\bigoplus_{\lambda \in \Lambda} R_{\lambda}^{1}\right)=0$. By an argument like that used for slender abelian groups(in which the identity elements of the $R_{\lambda}^{1}$ play a key role) it follows that $f=0$ and then $\prod_{\lambda \in \Lambda} R_{\lambda} \in U(S)$. Since $S$ is simple with identity, $U(S)$ is hereditary, and hence, as $\prod_{\lambda \in \Lambda} R_{\lambda} \triangleleft \prod_{\lambda \in \Lambda} R_{\lambda}^{1}, \prod_{\lambda \in \Lambda} R_{\lambda}$ is in $U(S)$ also.

Example 6.7. Both $\mathbb{Q}$ and $\mathbb{R}$ are slender (see [36]; the result there relies on $[11])$, but $\mathbb{C}, M_{2}(\mathbb{R})$ and $M_{2}(\mathbb{Q})$ are not. 
We do not know how $U\left(M_{2}(\mathbb{R})\right)$ or $U\left(M_{2}(\mathbb{Q})\right)$ fare with respect to products, but since $\mathbb{C}$ is an ultraproduct of the algebraic closures of the fields $\mathbb{Z}_{p}$ (see, e.g., [73]), we do have the following.

Proposition 6.8. $U(\mathbb{C})$ is not closed under countable products.

Things are different for associative algebras over a field. Using a result of Bergman and Nahlus ([9], Theorem 11) we get

Example 6.9. If $\mathcal{M}$ is a class of idempotent simple algebras of countable dimension over an infinite field $K$ and if $K$ is non-measurable, then in any variety of $K$-algebras containing $\mathcal{M}, U(\mathcal{M})$ is closed under non-measurable products.

For some variations, see Theorem 9 of [10].

\section{$7 \quad$ Ring results related to regularity}

Let $\rho, \sigma$ be integer polynomial functions defined on a ring $R$ with values in a unital extension. Then $R$ is said to be $(\rho ; \sigma)$ - regular if for each $a \in R$ there exists an element $b \in R$ such that $a=\rho(a) b \sigma(a)$. For every $\rho, \sigma$, the class $\mathcal{R}_{\rho \sigma}$ of $(\rho ; \sigma)$-regular rings is a radical class. The idea seems to be due to McKnight and is studied in [42], [58], [59],[60]. In these papers the polynomial functions are called $p, q$.

If $R_{\lambda} \in \mathcal{R}_{\rho \sigma}$ for all $\lambda \in \Lambda$ and $\left(a_{\lambda}\right)_{\Lambda} \in \prod_{\lambda \in \Lambda} R_{\lambda}$, then for each $\lambda$ there is a $b_{\lambda}$ in $R_{\lambda}$ such that $a_{\lambda}=\rho\left(a_{\lambda}\right) b_{\lambda} \sigma\left(a_{\lambda}\right)$, whence

$\left(a_{\lambda}\right)_{\Lambda}=\left(\rho\left(a_{\lambda}\right) b_{\lambda} \sigma\left(a_{\lambda}\right)\right)_{\Lambda}=\left(\rho\left(a_{\lambda}\right)\right)_{\Lambda}\left(b_{\lambda}\right)_{\Lambda}\left(\sigma\left(a_{\lambda}\right)\right)_{\Lambda}$

$=\rho\left(\left(a_{\lambda}\right)_{\Lambda}\right)\left(b_{\lambda}\right)_{\Lambda} \sigma\left(\left(a_{\lambda}\right)_{\Lambda}\right)$.

Thus we have

Example 7.1. The radical class $\mathcal{R}_{\rho \sigma}$ is closed under products for every $\rho, \sigma$.

Examples of radical classes $\mathcal{R}_{\rho \sigma}$ include

- the regular rings $(\rho(x)=x=\sigma(x))[12]$;

- the quasiregular rings $(\rho(x)=x+1, \sigma(x)=1)$;

- the $D$-regular rings $(\rho(x)=1, \sigma(x)=x)[16]$;

- the strongly regular rings $\left(\rho(x)=x^{2}, \sigma(x)=1\right)$ [76].

(The references are to papers where the concept was introduced or first recognized as defining a radical class.)

A rather degenerate example, but which we shall discuss again later, is obtained when for some $n \in \mathbb{Z}$ we take $\rho(x)=n, \sigma(x)=1$. In this case $\mathcal{R}_{\rho \sigma}=\mathcal{D}_{S}^{*}$, the class of $S$-divisible rings, where $S$ is the set of prime divisors of $n$. 
The class of strongly regular rings is a variety of type $\langle 2,2,0,1,1\rangle$ where as well as the ring operations we have a unary operation ', and the identities $x^{2} x^{\prime} \approx x \approx x^{\prime} x^{2} ;\left(x^{\prime}\right)^{\prime} \approx x$. The class of regular rings is not a variety, e.g. because it is not closed under equalizers [69]. Nevertheless, if $R$ is a regular ring and $a \in R$ then there is an element $a^{\prime} \in R$ such that $a=a a^{\prime} a$ and $a^{\prime}=a^{\prime} a a^{\prime}$. (Note that if $a=a b a$ it doesn't follow that $b=b a b$, but if we take $c=b a b$, we get $a=a c a$ and $c=c a c$.) Although $a^{\prime}$ is not uniquely determined by $a$, if one chooses an $a^{\prime}$ for each $a$, there results a unary operation satisfying the identities $x \approx x x^{\prime} x$ and $x^{\prime} \approx x^{\prime} x x^{\prime}$. A procedure like this was first used by Hall [44] and Kad'ourek and Szendrei [54] in regular semigroups. For regular rings it has been used by Herrmann and Semenova [46].

An $e$-variety of regular rings is a non-empty class closed under products, homomorphic images and regular subrings. This is equivalent to being a variety $\mathcal{V}$ of regular rings with an operation ' as described with the additional condition that if $R$ is regular and ' and * different allowable operations on $R$, then

$$
\left(R,{ }^{\prime}\right) \in \mathcal{V} \Leftrightarrow\left(R,{ }^{*}\right) \in \mathcal{V} \ldots \ldots \ldots \ldots \ldots \ldots(\dagger)
$$

Varieties of strongly regular rings, e.g commutative, additively torsion-free (or, equivalently, divisible) and the class of strongly regular rings itself, are $e$-varieties of regular rings.

For any class $\mathcal{K}$ of regular rings, let $\mathcal{K}^{\prime}$ be the class of structures

$$
\left\{\left(R,+, \cdot, 0,-,^{\prime}\right):(R,+, \cdot, 0,-) \in \mathcal{K}\right\},
$$

where' satisfies the identities $x \approx x x^{\prime} x, x^{\prime} \approx x^{\prime} x x^{\prime}$. The restriction of the correspondence $\mathcal{K} \mapsto \mathcal{K}^{\prime}$ defines a bijection from $e$-varieties of regular rings to those varieties of structures with the additional unary operation which satisfy $(\dagger)$. See [46] for this.

Varieties which are closed under extensions are product-closed radical classes, and for associative rings, such varieties consist of regular rings. Our next result generalizes this.

Theorem 7.2. Every e-variety of regular rings is a product-closed radical class of associative rings.

Proof. Let $\mathcal{V}$ be an $e$-variety of regular rings. If $I \triangleleft A \in \mathcal{V}$ then $A / I \in \mathcal{V}$. Let $\left\{I_{\lambda}: \lambda \in \Lambda\right\}$ be a chain of ideals of a ring with each $I_{\lambda} \in \mathcal{V}$. If $x \in I_{\lambda}$, $x=x x^{\prime} x$ and $x^{\prime}=x^{\prime} x x^{\prime}$, then $x^{\prime} \in I_{\lambda}$. This means that $I_{\lambda}$ is in the variety $\mathcal{V}^{\prime}$. But then $\bigcup_{\lambda \in \Lambda} I_{\lambda} \in \mathcal{V}^{\prime}$ so $\bigcup_{\lambda \in \Lambda} I_{\lambda} \in \mathcal{V}$. It remains to be shown that $\mathcal{V}$ is closed under extensions.

If $T$ is regular and subdirectly irreducible with heart $H \in \mathcal{V}$, then as $T$ is in the $e$-variety generated by $H$ ([46], Corollary 18), also $T \in \mathcal{V}$. Since obviously 
if a subdirectly irreducible ring is in $\mathcal{V}$ then so, being regular, is its heart, we conclude that if $T$ is regular and subdirectly irreducible with heart $H$, then

$T$ is in $\mathcal{V}$ if and only if $H$ is in $\mathcal{V}$.

Now let $R$ be in $V$ and let $S$ be a regular essential extension of $R$. Then $R$ has ideals $J_{i}$ with $\bigcap J_{i}=0$ and each $R / J_{i}$ subdirectly irreducible (and in $\mathcal{V}$ ). By regularity, $J_{i} \triangleleft S$ for each $i$ and so $S$ is a subdirect product of the $S / J_{i}$. For each $i$ let $K_{i}$ be an ideal of $S$ such that $K_{i} \cap R=J_{i}$ and $K_{i}$ is maximal for this. Then $K_{i} / J_{i} \triangleleft S / J_{i}, K_{i} / J_{i} \cap R / J_{i}=0$ and $K_{i} / J_{i}$ is maximal for this. Hence

$$
R / J_{i} \cong\left(R / J_{i}+K_{i} / J_{i}\right) /\left(K_{i} / J_{i}\right) \triangleleft^{\bullet}\left(S / J_{i}\right) /\left(K_{i} / J_{i}\right) \cong S / K_{i} .
$$

(Here $\triangleleft^{\bullet}$ indicates an essential ideal.) Let $H_{i}$ be the heart of $R / J_{i}$ for each $i$. Then $H_{i}$ is regular and simple and therefore isomorphic to an ideal of $S / K_{i}$. It follows that $S / K_{i}$ is subdirectly irreducible with heart isomorphic to $H_{i}$. Since $R / J_{i}$ is subdirectly irreducible and in $\mathcal{V}$, it follows from $\left(^{*}\right)$ that $S / J_{i} \in \mathcal{V}$. Then $S$ is a subdirect product of the $S / J_{i}$ and hence a regular subring of their product, so $S \in \mathcal{V}$. This shows that $\mathcal{V}$ is closed under regular essential extensions.

Now if $C \triangleleft D$ with $C, D / C \in \mathcal{V}$ there is an ideal $M$ of $D$ with $M \cap C=0$ and $M$ maximal for this. Then $C \cong(C+M) / M \triangleleft \bullet D / M$ and $D / M$ is regular, as $D$ is regular $(C$ and $D / C$ being so) whence $D / M \in \mathcal{V}$. Since $D$ is a subdirect product of $D / C$ and $D / M$, both of which are in $\mathcal{V}$, so also $D$ is in $\mathcal{V}$. Thus $\mathcal{V}$ is closed under extensions and the proof is complete.

Example 7.3. Here are some further examples of $e$-varieties of regular rings and hence of product closed radical classes of associative rings.

- The class of regular rings in any variety of associative rings (see also [29]). Note that this is the same as the class of hereditarily idempotent rings in a variety [4].

- The torsion-free regular rings.

- The regular rings of characteristic $p$ where $p$ is a fixed prime.

- Any variety of strongly regular rings, e.g. all strongly regular rings, the torsion-free ones, those of characteristic $p$ and the semi-simple-radical classes.

For $n \in \mathbb{Z}^{+}$let $\mathcal{R}_{n}$ denote the class of regular rings in which $a^{n}=0$ for every nilpotent element $a$ (so $\mathcal{R}_{1}$ is the class of strongly regular rings). By Theorem 1.6 of [30]

- $\mathcal{R}_{n}$ is an $e$-variety of regular rings for every $n$.

By [30], Theorem 4.4, every product closed radical class of regular rings with all primitive homomorphic images artinian is contained in some $\mathcal{R}_{n}$. On 
the other hand, by [46], Corollary 17, every $e$-variety of regular rings is generated as an $e$-variety by its artinian members.

Let us note that the class of torsion regular rings is a radical class closed under regular subrings, but not products.

The class of regular rings, being hereditary and homomorphically closed, can serve as a universal class for radical theory. It is well known (proved in [5]) that when a hereditary radical class is used as a universal class, all its radical classes remain so when viewed in the ambient universal class. The same cannot be said for semi-simple classes: in regular rings the class of torsion-free regular rings is the semi-simple class for the torsion regular rings. It is not a semisimple class of associative rings, however; e.g. for a field $K$ of characteristic $0, K[X]$ is a subdirect product of copies of $K$.

\section{Modules}

In this section, all rings have identities and "module" means "unital left module"; $\operatorname{Mod}(R)$ denotes the category of $R$-modules.

The hereditary product-closed radical classes of modules over a ring $R$ are the classes $\nu_{I}=\{M: I M=0\}$ for idempotent ideals $I$ of $R$, as proved by Jans [51], and are of course varieties and thus semi-simple radical classes (and generally called TTF-classes). For hereditary and splitting properties of the two radicals associated with an idempotent ideal, see Azumaya [6], Nicolás and Saorín [61].

For hereditary radical classes we have the following oft-proved result [1], [18], [27], [71].

Example 8.1. If $R$ is right perfect then there are only finitely many hereditary radical classes, each is closed under products and (apart from $\{0\}$ ) each is the lower radical class defined by a set of simple modules.

The converse of this result is false, as was proved by Dlab [18]. We'll now show this, but using a more convenient (and, for radical-theorists, more familiar) ring.

Let $A$ be an algebra over a field $K$ with a basis $\left\{e_{a}: a \in[0,1]\right\}$ with multiplication given by

$$
e_{a} e_{b}=\left\{\begin{aligned}
e_{a+b} & \text { if } a+b \leq 1 \\
0 & \text { if } a+b>1
\end{aligned}\right.
$$

(Thus $A$ is obtained from the Zassenhaus algebra by the adjunction of an identity $e_{0}$ and an annihilating basis element $e_{1}$ ). Let $J$ be the subspace 
spanned by $\left\{e_{a}: a>0\right\}$. Then $J$ is nil and $A / J \cong K$ so $J=\mathcal{J}(A)$. If $j \in J$ and $\alpha \in K \backslash\{0\}$ let $\ell \in J$ be such that $\alpha^{-1} j \circ \ell=0$. Then $\left(\alpha e_{0}+j\right)\left(\alpha^{-1} e_{0}+\right.$ $\alpha^{-1} \ell$ ) $=e_{0}+\ell \circ \alpha^{-1} j=e_{0}$ so $\alpha e_{0}+j$ is a unit. (Here $\circ$ is the circle operation used in the definition of quasiregularity.) Hence all proper ideals are contained in $J$. If $I \triangleleft A$ and $I \subsetneq J$ then $I$ is nilpotent [17].

Let $\Phi$ be an idempotent topologizing filter of ideals of $A, \mathcal{R}$ the corresponding hereditary radical class of $A$-modules. If $I \in \Phi$ then $A / I \in \mathcal{R}$. Also for each $i \in I$ we have $I\left(i+I^{2}\right)=0$ so $\left(0: i+I^{2}\right) \supseteq I \in \Phi$ and it follows that $I / I^{2} \in \mathcal{R}$. Now $\left(A / I^{2}\right) /\left(I / I^{2}\right) \cong A / I \in \mathcal{R}$, so $\bar{A} / I^{2} \in \mathcal{R}$ and thus $I^{2} \in \Phi$. In the same way $\Phi$ contains $I^{4}, I^{8}, \ldots$, so if $I$ is nilpotent we have $0 \in \Phi$ and thus $\mathcal{R}=\operatorname{Mod}(A)$. If $\Phi$ contains no nilpotent ideals then $\Phi=\{J, A\}$ or $\{A\}$ and only the former is of interest. In this case, as $J$ is idempotent, $\mathcal{R}=\{M: J M=0\}$.

Example 8.2. The hereditary radical classes of $A$-modules, for $A$ as defined, are $\operatorname{Mod}(A),\{M: J M=0\}$ and $\{0\}$, all of which are closed under products. As $\mathcal{J}(A)=J=J^{2}, A$ is not perfect.

One might expect to see some results and examples for modules occurring as generalizations or imitations of results and examples for abelian groups. Of course we have the " $A$-radicals", the classes of modules with $S$-divisible additive groups, in particular the modules with divisible groups. A more interesting analogue of this last would be the class of injective modules, but while the class of injective $R$-modules is always product closed, it is closed under direct sums if and only if $R$ is noetherian. (This well known result is attributed to Bass by Faith [22].) Since we also need homomorphic closure, we have

Example 8.3. The class of injective $R$-modules is a (necessarily product closed) radical class if and only if $R$ is noetherian and hereditary.

There is a version of slenderness for modules too: an $R$-module $M$ is slender if every $R$-module homomorphism from $R^{\omega}$ to $M$ takes almost all copies of $R$ to 0 . As with abelian groups (cf. [15]) we have

Theorem 8.4. For every class $\mathcal{S}$ of slender $R$-modules, $U(\mathcal{S})$ is closed under non-measurable products.

Corollary 8.5. If a ring $R$ is slender as an $R$-module then in $\operatorname{Mod}(R), U(R)$ is closed under non-measurable products.

Of course $\mathbb{Z}$ is a slender $\mathbb{Z}$-module. More such rings are given in the following examples. 
Example 8.6. - If an integral domain $R$ has infinitely many maximal ideals and every infinite set of maximal ideals has zero intersection, then $R$ is a slender $R$-module. In particular a Dedekind domain $A$ with infinitely many prime ideals is a slender $A$-module [2],[3]; see also [56].

- Polynomial rings are slender as modules. [65]

- If $R$ is a slender $R$-module, then $M_{n}(R)$ is a slender $M_{n}(R)$-module for each $n$. [65]

We don't know anything about a converse to Corollary 8.5, but the ring $I_{p}$ of $p$-adic integers is not slender (cf. [65], Proposition 2.4) and its upper radical class is not closed under non-measurable products.

Example 8.7. In $\operatorname{Mod}\left(I_{p}\right), U\left(I_{p}\right)$ is not closed under countable products.

Proof. Every non-zero torsion-free reduced $I_{p}$-module has a direct summand isomorphic to $I_{p}$. (See, e.g. [55], p.53.) Hence $U\left(I_{p}\right)$ is the class of extensions of torsion modules by divisible torsion-free modules. Let $M=\prod_{n \in \mathbb{Z}^{+}} I_{p} / p^{n} I_{p}$ and let $a_{n}$ be a generator of $I_{p} / p^{n} I_{p}$ for each $n$. If $\left(a_{n}\right)-p\left(r_{n} a_{n}\right)$ were in the torsion submodule $T$ of $M$ for some $r_{n} \in I_{p}$ there would be a non-zero $s \in I_{p}$ for which $s\left(\left(a_{n}\right)-p\left(r_{n} a_{n}\right)\right)=0$ and thus $\left(s-s p r_{n}\right) a_{n}=0$ for each $n$. But then we'd have $p^{n} \mid s\left(1-p r_{n}\right)$ for all $n$. But $s=t p^{k}$ for some unit $t$ and some $k$, so this would require $p^{n} \mid t p^{k}\left(1-p r_{n}\right)$ for all $n$ and thus $p^{n-k} \mid\left(1-p r^{n}\right)$ for all $n>k$. This is impossible. Hence there exists no such $\left(r_{n} a_{n}\right)$ so $\left(a_{n}\right)+T$ has zero $p$-height in $M / T$ which is therefore not divisible and so has a non-zero torsion-free reduced homomorphic image. Hence $I_{p}$ is a homomorphic image of $M / T$ so $M \notin U\left(I_{p}\right)$.

\section{Essential Extensions}

It was shown by Loi [57] that every essentially closed radical class of associative rings is closed under products and is strongly hereditary and thus is a variety and a semi-simple class. The non-trivial semi-simple radical classes define upper radical classes which are special and hence hereditary so these radical semi-simple classes are essentially closed. Thus we have

Example 9.1. A radical class of associative rings is essentially closed if and only if it is a variety.

For arbitrary non-associative rings Loi's proof works equally well: an essentially closed radical class is a variety. There are no semi-simple radical classes here [28] and probably no radical classes which are varieties. It's not clear which other classes of rings satisfy Loi's theorem: the proof only extends 
to classes in which (i) every ring has a unital essential extension and (ii) a certain construction is possible. However Veldsman [77] has obtained some results for nearrings.

Example 9.2. If $\mathcal{R}$ is an essentially closed radical class of nearrings such that all members of $\mathcal{R}$ are zero-symmetric and each $N \in \mathcal{R}$ can be embedded in a zero-symmetric nearring with zero annihilator, then $\mathcal{R}$ is a variety.

(Cf. [77], 17. Theorem.)

Being essentially closed is thus a very restrictive condition for radical classes in some settings. On the other hand for modules there is nothing really dramatic about it; for instance all radical classes of abelian groups are essentially closed.

Note also that semi-simple radical classes need not be essentially closed. Let $R$ be a ring with identity. If $\mathcal{R}$ is a semi-simple radical class of $R$-modules then $\mathcal{R}=\{M: I M=0\}$ for some idempotent ideal $I$ of $R$. A result of Azumaya ([6], Theorem 6), says that $\mathcal{R}$ is essentially closed if and only if $R / I$ is a flat right $R$-module.

We examine essentially closed radical classes in one more case: groups. Here there are no non-trivial varieties which are radical classes. It turns out that there are no non-trivial essentially closed radical classes either.

Lemma 9.3. If $G$ is a group and $H \triangleleft G$, its centralizer $C(H)$ is also a normal subgroup.

Proof. If $k \in C(H)$ and $a \in G$, then for all $h \in H$ we have $\left(a k a^{-1}\right) h=$ $a k a^{-1} h\left(a a^{-1}\right)=a k\left(a^{-1} h a\right) a^{-1}=a\left(a^{-1} h a\right) k a^{-1}=h\left(a k a^{-1}\right)$.

Let $A, B$ be groups, $\varphi: B \rightarrow A u t(A)$ a homomorphism. Let $A \rtimes_{\varphi} B$ denote the associated semidirect product, and identify $A$ with its natural copy in the group $A \rtimes_{\varphi} B$. If $(a, b) \in C(A)$ then for all $x \in A$ we have $(a, b)(x, e)=(a \varphi(b)(x), b)$ and $(x, e)(a, b)=(x \varphi(e)(a), b)$ so $a \varphi(b)(x)=x a$ and thus $\varphi(b)(x)=a^{-1} x a$. Conversely, if $\varphi(b)(x)=a^{-1} x a$ for all $x \in A$ then $(a, b)(x, e)=\left(a \cdot a^{-1} x a, b e\right)=(x a, b)=(x, e)(a, b)$. Thus

$$
C(A)=\left\{(a, b): \varphi(b)(x)=a^{-1} x a \forall x \in A\right\} .
$$

Theorem 9.4. For groups $A, B$ with $|A| \geq 2$ let $A \nmid B$ denote the left regular restricted wreath product. Then $A^{(B)} \triangleleft^{\bullet} A$ 々 $B$.

(Here $A^{(B)}$ is the restricted direct product (direct sum).)

Proof. For the homomorphism $\chi: B \rightarrow A u t\left(A^{(B)}\right)$ for which $\chi(b)$ takes $\left(a_{y}\right)_{B}$ to $\left(c_{y}\right)_{B}$, where $a_{y}=c_{b y}$, we have $A$ $B=A^{(B)} \rtimes_{\chi} B$. Let $d$ be in $A$ with $d \neq e$ 
and $b \in B$ and let $d_{y}=d$ if $y=b$ and $e$ otherwise. Then $\chi(b)\left(\left(d_{y}\right)_{B}\right)=\left(f_{y}\right)_{B}$ where $e=d_{e}=f_{b}$ and $d_{b}=d \neq e$ if $b \neq e$. Thus $\left(f_{y}\right)_{B}$ is not a conjugate of $\left(d_{y}\right)_{B}$ in $A^{(B)}$, so that $\chi((b)$ can't be an inner automorphism. Hence $C\left(A^{(B)}\right) \subseteq A^{(B)}$. If now $\left.K \triangleleft A\right\} B$ and $K \cap A^{(B)}=\{e\}$, then for all $k \in K, x \in$ $A^{(B)}$ we have $[k, x]=k\left(x k^{-1} x^{-1}\right) \in K$ and $[k, x]=\left(k x k^{-1}\right) x^{-1} \in A^{(B)}$. But then $K \subseteq C\left(A^{(B)}\right) \subseteq A^{(B)}$ and so $K=\{e\}$.

Corollary 9.5. There are no non-trivial essentially closed radical classes of groups.

Proof. Let $\mathcal{R}$ be an essentially closed radical class of groups containing a group $A$ with at least two elements. For every group $B$ we have $A^{(B)} \in \mathcal{R}$ and $A^{(B)} \triangleleft^{\bullet} A$ 乙 $B$ so $\mathcal{R}$ contains $A$ 乙 $B$ and hence also $B$.

Thus, vacuously, we have a result analogous to Loi's theorem.

Example 9.6. A radical class of groups is essentially closed if and only if it is a variety.

\section{Inverse limits}

If $\Gamma$ is a directed index set, $\left\{A_{\gamma}: \gamma \in \Gamma\right\}$ a set of rings or groups or modules and so on, and whenever $\gamma \geq \delta$ there is a homomorphism $\pi_{\delta}^{\gamma}: A_{\gamma} \rightarrow A_{\delta}$ such that

$\pi_{\gamma}^{\gamma}$ is the identity homomorphism for every $\gamma$ and

$\pi_{\epsilon}^{\delta} \circ \pi_{\delta}^{\gamma}=\pi_{\epsilon}^{\gamma}$ whenever $\gamma \geq \delta \geq \epsilon$,

the inverse limit $\lim _{\leftarrow} A_{\gamma}$ of this system is

$$
\left\{\left(a_{\gamma}\right)_{\Gamma} \in \prod A_{\gamma}: \pi_{\delta}^{\gamma}\left(a_{\gamma}\right)=a_{\delta} \text { whenever } \gamma \geq \delta\right\} .
$$

Alternatively $\lim _{\leftarrow} A_{\gamma}$ is a solution to a universal mapping problem: for each $\delta \in \Gamma$ there is a homomorphism $\pi_{\delta}: \lim _{\leftarrow} A_{\gamma} \rightarrow A_{\delta}$ (namely the projection) such that $\pi_{\delta}^{\gamma} \circ \pi_{\gamma}=\pi_{\delta}$ whenever $\gamma \geq \delta$ and $\lim _{\leftarrow} A_{\gamma}$ is universal for this.

Adopting the latter point of view we can show that if $\prod_{\lambda \in \Lambda} A_{\lambda}$ is a direct product, $\Gamma$ is the set of finite subsets of $\Lambda$, ordered by inclusion and for $E \subseteq F$, $\pi_{E}^{F}$ is the obvious projection, then $\lim _{\leftarrow} \bigoplus_{\lambda \in F} A_{\lambda} \cong \prod_{\lambda \in \Lambda} A_{\lambda}$. (See, e.g., [23], Vol. 1, p.62.)

In this case, each $\pi_{E}^{F}$ is surjective. We shall call an inverse limit arising from a system of surjective homomorphisms a surjective inverse limit. From the above we see that 
If a radical class is closed under surjective inverse limits then it is closed under products.

(Inverse limits in general include, among other things, intersections of subobjects, which have little connection with our subject.)

Inverse limits of countable surjective inverse systems of regular rings are regular [66], Proposition 1.1. Hence, as an inverse limit of regular rings is a subring of a direct product of regular rings, every $e$-variety of regular rings is closed under such inverse limits.

Example 10.1. All $e$-varieties of regular rings (in particular the class of all regular rings) are closed under inverse limits of countable surjective inverse systems.

Example 10.2. Inverse limits of quasiregular rings are quasiregular [49], i.e. the Jacobson radical class is closed under surjective inverse limits.

Radical classes $\mathcal{R}_{\rho \sigma}$ need not be closed under surjective inverse limits; by adapting an example of Bergman ([8], Example 10) we can get

Example 10.3. For a prime $p$, the class $\mathcal{D}_{p}$ of $p$-divisible abelian groups is not closed under limits of countable surjective inverse systems and hence neither is the class $\mathcal{D}_{p}^{*}$ of $p$-divisible associative rings.

For each $n \in \mathbb{Z}^{+}$let

$$
M_{n}=\underbrace{\mathbb{Q}(p) \oplus \mathbb{Q}(p) \oplus \ldots \oplus \mathbb{Q}(p)}_{n-1} \oplus \mathbb{Z}\left(p^{\infty}\right) \oplus \mathbb{Z}\left(p^{\infty}\right) \oplus \ldots .
$$

For $n \geq m$ let $\pi_{m}^{n}: M_{n} \rightarrow M_{m}$ be defined componentwise, with each component being the identity map $\mathbb{Q}(p) \rightarrow \mathbb{Q}(p)$, the identity map $\mathbb{Z}\left(p^{\infty}\right) \rightarrow \mathbb{Z}\left(p^{\infty}\right)$ or the natural map $\mathbb{Q}(p) \rightarrow \mathbb{Z}\left(p^{\infty}\right)$ as appropriate. Then $\lim _{\leftarrow} M_{n} \cong\left\{\left(x_{n}\right) \in\right.$ $\mathbb{Q}(p)^{\omega}: x_{n} \in \mathbb{Z}$ for almost all $\left.n\right\}$. Clearly this is not $p$-divisible. Taking zerorings we get the result for rings.

\section{Some Problems Concerning Smallest Radical Classes}

It is clear that an intersection of product closed radical classes is a product closed radical class, so each class $\mathcal{M}$ is contained in a smallest product closed radical class which we'll call $L_{\Pi}(\mathcal{M})$. Nothing seems to be known about this: there don't even seem to be any known non-trivial examples of classes $L_{\Pi}(\mathcal{M})$. Here are some problems which seem worthy of attention.

1. Find a construction for $L_{\Pi}(\mathcal{M})$.

2. What is $L_{\Pi}(\mathcal{M})$ (in asociative rings or elsewhere) when $\mathcal{M}$ is the class of zerorings? 
3. What is $L_{\Pi}(\mathcal{M})$ in groups when $\mathcal{M}$ is the class of abelian groups?

4. If $\mathcal{M}$ is hereditary, must $L_{\Pi}(\mathcal{M})$ be hereditary?

5 . Investigate product closed special radical classes.

In connection with 2., note that Example 6.5 provides a radical class much smaller than $\mathcal{J}$ which contains the zerorings and is closed under non-measurable products. On the other hand, every radical class containing all zerorings must contain, for any prime $p$, all rings $p \mathbb{Z}_{p^{n}}$ and the product of these is non-nil.

Concerning 3. we note a result of Phillips [67], Theorem 4, which states that no class of groups which contains all finite solvable groups and is contained in the lower radical class defined by the locally nilpotent groups can be closed under products. Thus a product closed radical class containing all abelian groups can't be contained in the lower radical class defined by the locally nilpotent groups.

Analogously we can ask about the smallest radical class $L_{\leftarrow}(\mathcal{M})$ which contains $\mathcal{M}$ and is closed under surjective inverse limits. We get five more open problems by substituting inverse limits and $L_{\leftarrow}(\mathcal{M})$ for direct products and $L_{\Pi}(\mathcal{M})$ in 1.-5.

Bergman [7] observes that the power series over a field $K$ in two noncommuting indeterminates $X$ and $Y$ with zero $X$ - and $Y$-free term form a ring which is a surjective inverse limit of nilpotent rings, and the Sa̧siadaCohn simple quasiregular ring [72] is an ideal of a homomorphic image of such a ring. Thus every hereditary radical class which contains all zerorings and is closed under surjective inverse limits must contain the Sąsiada-Cohn ring. Also $\mathcal{J}$ is closed under surjective inverse limits as we have noted (Example 10.2).

\section{References}

[1] J. S. Alin and E. P. Armendariz, TTF classes over perfect rings, J. Austral. Math. Soc. 11 (1970), 499-503.

[2] D. Allouch, Modules maigres, Thesis, Faculté des Sciences de Montpellier, 1969-70.

[3] D. Allouch, Les modules maigres, C. R. Acad. Sci. Paris Sér. A-B 272 (1971), A517-A519.

[4] E. P. Armendariz and J. W. Fisher, Regular P.I. rings, Proc. Amer. Math. Soc. 39 (1973), 247-251.

[5] V. I. Arnautov and M. I. Vodinchar, Radikaly topologicheskikh kolets, Mat. Issled. 3 (1968), No. 2, 31-61. 
[6] G. Azumaya, Some properties of TTF-classes, Proc. Conference on Orders, Group Rings and Related Topics (Ohio State Univ., Columbus, 1972) pp. 72-83. Berlin: Springer, 1973.

[7] G. M. Bergman, Homomorphic images of pro-nilpotent algebras, preprint, arXiv: 0912.0020v1 [math.RA] 30 Nov 2009

[8] G. M. Bergman, Every module is an inverse limit of injectives, preprint, arXiv:1104:3173v2 [math.RA] 16 Aug 2011

[9] G. M. Bergman and N. Nahlus, Homomorphisms on infinite direct product algebras, especially Lie algebras, J. Algebra 333 (2011), 67-104.

[10] G. M. Bergman and N. Nahlus, Linear maps on $k^{I}$, and homomorphic images of infinite direct product algebras, J. Algebra 356 (2012), 257-274.

[11] R. Börger and M. Rajagopalan, When do all ring homomorphisms depend on only one co-ordinate?, Arch. Math. 45 (1985), 223-228.

[12] B. Brown and N. H. McCoy, The maximal regular ideal of a ring, Proc. Amer. Math. Soc. 1 (1950), 165-171.

[13] C. Casacuberta, J. L. Rodríguez and D. Scevenels, Singly generated radicals associated with varieties of groups Groups St. Andrews 1997 in Bath, I, pp. 202-210. Cambridge: Cambridge Univ. Press, 1999.

[14] A. L. S. Corner and R. Göbel, Radicals commuting with cartesian products, Arch. Math. 71 (1998), 341-348.

[15] R. Dimitrić, Slenderness in abelian categories, Abelian Group Theory (Proc., Honolulu 1982/83), pp. 375-383. Berlin etc.: Springer, 1983.

[16] N. Divinsky, D-regularity, Proc. Amer. Math. Soc. 9 (1958), 62-71.

[17] N. Divinsky, Unequivocal rings, Canad. J. Math. 27 (1975), 679-690.

[18] V. Dlab, A characterization of perfect rings, Pacific. J. Math. 33 (1970), 79-88.

[19] M. Dugas and R. Göbel, On radicals and products, Pacific. J. Math. 118 (1985), 79-104.

[20] K. Eda, A characteriztion of $\aleph_{1}$-free abelian groups and its application to the Chase radical, Israel J. Math. 60 (1987), 22-30. 
[21] K. Eda, Cardinality restrictions on preradicals, Abelian Group Theory (Proc. 1987 Perth Conference), pp. 277-283. Providence: American Mathematical Society, 1989.

[22] C. Faith, Lectures on Injective Modules and Quotient Rings, Berlin etc.: Springer, 1967.

[23] L. Fuchs, Infinite Abelian Groups Vols. I and II, New York and London: Academic Press, 1970, 1973.

[24] B. J. Gardner, Torsion classes and pure subgroups, Pacific J. Math. 33 (1970), 109-116.

[25] B. J. Gardner, Some closure properties for torsion classes of abelian groups, Pacific. J. Math. 42 (1972), 45-61.

[26] B. J. Gardner, Two notes on radicals of abelian groups, Comment. Math. Univ. Carolinae 13 (1972), 419-430.

[27] B. J. Gardner, Some aspects of T-nilpotence II: Lifting properties over T-nilpotent ideals, Pacific J. Math. 59 (1975), 445-453.

[28] B. J. Gardner, Semi-simple radical classes of algebras and attainability of identities, Pacific. J. Math. 61 (1975), 401-416.

[29] B. J. Gardner, Polynomial identities and radicals, Compositio. Math. 35 (1977), 269-279.

[30] B. J. Gardner, Radical classes of regular rings with artinian primitive images, Pacific. J. Math. 99 (1982), 337-349.

[31] B. J. Gardner, When are radical classes of abelian groups closed under direct products? Algebraic Structures and Applications (Proc. First Western Australian Conference on Algebra, 1980), pp. 87-99. New York: Marcel Dekker, 1982.

[32] B. J. Gardner, Radicals and varieties, Radical Theory (Proc. Eger, 1982), pp. 93-133. Amsterdam etc.: North-Holland, 1985.

[33] B. J. Gardner, Radical Theory, Harlow: Longman, 1989.

[34] B. J. Gardner, Some cardinality conditions for ring radicals, Quaestiones Math. 15 (1992), 27-37.

[35] B. J. Gardner, On product-closed radical classes of abelian groups, Buletinul Acad. Ştiinţe Repub. Moldova Matematica 2(30) (1999), 33-52. 
[36] B. J. Gardner, Slenderness for rings, Vietnam J. Math. 27 (1999), 345361.

[37] B. J. Gardner, There isn't much duality in radical theory, Algebra Discrete Math. 2007, No. 3, 59-66.

[38] B. J. Gardner and R. Wiegandt, Radical Theory of Rings, New YorkBasel: Marcel Dekker, 2004.

[39] J. Gismatullin and A. Muranov, A remark on groups without finite quotients, preprint, arXiv:0903.2536v3 [math.GR] 21 Mar 2010

[40] R. Göbel, Kartesische Produkte von Gruppen, Arch. Math. 26 (1975), 454-462.

[41] R. Göbel, On stout and slender groups, J. Algebra 35 (1975), 39-55.

[42] T. L. Goulding and A. H. Ortiz, Structure of semiprime $(p, q)$ radicals, Pacific J. Math. 37 (1971), 97-99.

[43] A. W. Hager and J. J. Madden, Algebraic classes of abelian torsion-free and lattice-ordered groups, Bull. Soc. Math. Grèce (NS), 25 (1984), 53-63.

[44] T. E. Hall, Identities for existence varieties of regular semigroups, Bull. Austral. Math. Soc. 40 (1989), 59-77.

[45] A. G. Heinicke, A note on lower radical constructions for associative rings, Canad. Math. Bull. 11 (1968), 23-30.

[46] C. Herrmann and M. Semenova, Existence varieties of regular rings and complemented modular lattices, J. Algebra 314 (2007), 235-251.

[47] G. Higman, B.H. Neumann and H. Neumann, Embedding theorems for groups, J. London Math. Soc. 24 (1949), 247-254.

[48] A. Hulanicki, The structure of the factor group of the unrestricted sum by the restricted sum of abelian groups, Bull. Acad. Polon. Sci. Sér. Sci. Math. Astronom. Phys. 10 (1962), 77-80.

[49] I. D. Ion, Radicalul limitei proiective de inele asociative, Stud. Cerc. Mat. 16 (1964), 1141-1145.

[50] A. M. Ivanov, Radikaly v kategorii vsekh abelevykh grupp bez krucheniya, Sibirsk. Mat. Zh. 20 (1979), 548-555.

[51] J. P. Jans, Some aspects of torsion, Pacific J. Math. 15 (1965),1249-1259. 
[52] T. Jech, Set Theory, New York etc.: Academic Press, 1978.

[53] Y. C. Jeon, N. K. Kim and Y. Lee, On fully idempotent rings, Bull. Korean Math. Soc. 47 (2010), 715-726.

[54] J. Kad'ourek and M. B. Szendrei, A new approach in the theory of orthodox semigroups, Semigroup Forum, 40 (1990), 257-296.

[55] I. Kaplansky, Infinite Abelian Groups, Ann Arbor: University of Michigan Press, 1969.

[56] E. L. Lady, Slender rings and modules, Pacific J. Math. 49 (1973), 397406.

[57] N. V. Loi, Essentially closed radical classes, J. Austral. Math. Soc. Ser. A 35 (1983), 132-142.

[58] J. D McKnight and G. L. Musser, Special $(p ; q)$ radicals, Canad. J. Math. 24 (1972), 38-44.

[59] G. L. Musser, Linear semiprime ( $p ; q)$ radicals, Pacific. J. Math. 37 (1971), 749-757.

[60] G. L. Musser, $[p ; q]$ radicals - a generalization of the Brown-McCoy radical, Tamkang J. Math. 5 (1974), 21-24.

[61] P. Nicolás and M. Saorín, Classification of split torsion torsionfree triples in module categories, J. Pure. Appl. Algebra 208 (2007), 979-988.

[62] R. J. Nunke, Slender groups, Acta Sci. Math (Szeged) 23 (1962), 67-73.

[63] R. J. Nunke, Purity and subfunctors of the identity, Topics in Abelian Groups (Proc. Symposium New Mexico State University, 1962), pp.121171. Chicago: Scott, Foresman, 1963.

[64] J. Olszewski, The Brown-McCoy radical is not closed under products, Comm. Algebra 22 (1994), 117-122.

[65] J. D. O'Neill, Slender modules over various rings, Indian J. Pure Appl. Math. 22 (1991), 287-293.

[66] G. K. Pedersen and F. Perera, Inverse limits of rings and multiplier rings, Math. Proc. Cambridge Philos. Soc. 139 (2005), 207-228.

[67] R. E. Phillips, On direct products of generalized solvable groups, Bull. Amer. Math. Soc. 73 (1967), 973- 975. 
[68] S. Pokutta and L. Strüngmann, The Chase radical and reduced products, J. Pure Appl. Algebra 211 (2007), 532-540.

[69] R. Raphael, Some remarks on regular and strongly regular rings, Canad. Math. Bull. 17 (1974/75), 709-712.

[70] A. H. Rhemtulla, A problem of bounded expressibility in free products, Proc. Cambridge Philos. Soc. 64 (1968), 573-584.

[71] E. A. Rutter, Torsion theories over semiperfect rings, Proc. Amer. Math. Soc. 34 (1972), 389-395.

[72] E. Sąsiada and P. M. Cohn, An example of a simple radical ring, J. Algebra 5 (1967), 373-377.

[73] H. Schoutens, The Use of Ultraproducts in Commutative Algebra Berlin etc.: Springer, 2010.

[74] S. Shelah, Infinite abelian groups, Whitehead problem and some constructions, Israel J. Math. 18 (1974), 243-256.

[75] A. L. Shmel'kin, Odno svoistvo poluprostykh klassov grupp, Sibirsk. Mat. Zh. 3 (1962), 950-951.

[76] T. Spircu, Radicalul strict regulat al unui inel, Stud. Cerc. Mat. 26 (1974), 751-754.

[77] S. Veldsman, Varieties and radicals of near-rings, Results Math. 24 (1993), 356-371.

Barry Gardner, University of Tasmania

Private Bag 37

Hobart, Tas. 7001, Australia

E-mail:gardner@hilbert.maths.utas.edu.au 